\title{
Oxygen Character in the Density of States as an Indicator of the Stability of Li-ion Battery Cathode Materials
}

M. D. Johannes ${ }^{1 *}$, Karen Swider-Lyons ${ }^{2}$, Corey T. Love ${ }^{2}$

${ }^{1}$ Center for Computational Materials Science, Naval Research Laboratory, Washington D.C.

${ }^{2}$ Alternative Energy Section, Chemistry Division, Naval Research Laboratory, Washington D.C.

*corresponding author

johannes@nrl.navy.mil

Keywords: Li-ion batteries; computation; oxygen stability; density functional theory

\begin{abstract}
Oxygen gas evolution from lithium battery cathode materials during charging to high voltages is a known mechanism leading to cathode instability and ultimately Li-ion batteries fires. We present the simple thesis that the tendency toward oxygen destabilization in metal-oxide materials used as lithium ion battery cathodes can be correlated to the amount of oxygen-derived weight in the partial density of states (PDOS) near the Fermi level $\left(\mathrm{E}_{\mathrm{F}}\right)$, which is easily calculable with Density Functional Theory (DFT). If the oxygen PDOS dominates the total DOS at $\mathrm{E}_{\mathrm{F}}$ at any point during delithiation (charging), electrons are withdrawn from the $\mathrm{O}$ rather than the $\mathrm{M}$ states, and the $\mathrm{O}^{2-}$ ions are oxidized to peroxide $\left(\mathrm{O}_{2}{ }^{2-}\right)$, and then often to $\mathrm{O}_{2}$ gas. A demonstration of the utility of this perspective is given using DFT to calculate the PDOS for compounds with a broad range of structures, transition metals and degrees of lithiation: $\mathrm{LiFePO}_{4}, \mathrm{Li}_{2} \mathrm{CuO}_{2}, \mathrm{LiCoO}_{2}, \mathrm{LiNiO}_{2}$, and $\mathrm{Li}_{2} \mathrm{RuO}_{3}$. The resulting computational spectra correlate well to the experimental stability in the modeled compounds. We conclude that the oxygen stability of cathode materials derives from their fundamental electronic structure as function of changing voltage (and lithiation).
\end{abstract}

\section{0}

\section{Introduction}

Lithium-ion batteries have been an enabling technology for mobile phones, computer tablets and laptops as their lightweight, compact formats reliably provide hours or even days of power to electronics, and 
consumers can easily replenish their energy from standard power outlets on electric grids. The most common active materials used in rechargeable batteries for portable electronics are a lithium cobalt oxide (nominally $\mathrm{LiCoO}_{2}$ ) cathode and a graphitic carbon anode, which are separated by a porous polymeric membrane and saturated in a carbonate-based electrolyte to yield a battery with a nominal voltage of $3.7 \mathrm{~V}$ vs $\mathrm{Li}^{+} / \mathrm{Li}^{0}$. The more recent application of $\mathrm{Li}$-ion batteries to electric and hybrid vehicles has raised the stakes for Li-ion battery technology, as the batteries have been integrated into high-voltage buses (300 V) in large formats. Such applications demand very high energy density, along with the capability for high power in a safe, low-cost battery. The demand for all of these qualities simultaneously has stimulated widespread research into alternative cathode materials

In metal-oxide cathode materials, a major safety and performance concern is $\mathrm{O}_{2}$ evolution, which can occur when the metal oxide is severely delithiated from overcharging or overheating. Such overcharging can occur in a multi-cell pack when one cell is an outlier in manufacturing, such that one cell is overcharged but the nominal pack voltage appears normal [1] but can also occur due to underlying and intrinsic materials instabilities at various stages of lithiation. Experimentally, Oxygen loss from cathode materials (e.g., $\mathrm{LiCoO}_{2}, \mathrm{LiNiO}_{2}$ and $\mathrm{LiMnO}_{2}$ ) was first identified by ex-situ thermogravimetric analysis [2], and has also been shown during the chemical extraction of $\mathrm{Li}^{+}$from delithiated $\mathrm{LiCoO}_{2}$, meant to simulate the effect of overcharging [3]. $\mathrm{O}_{2}$ release is not only exothermic, but liberated $\mathrm{O}_{2}$ can react with the solvent, SEI, and possibly the aluminum-metal current collectors. The loss of $\mathrm{O}_{2}$ with decreasing $\mathrm{Li}^{+}$content in $\mathrm{Li}_{\mathrm{x}} \mathrm{CoO}_{2}$ during overcharge has been attributed to the oxidation of the oxygen anions in the lattice to $\mathrm{O}_{2}$ rather than further oxidation of the cobalt cations [3]. A possible reaction for $\mathrm{O}_{2}$-gas loss from delithiated $\mathrm{Li}_{\mathrm{x}} \mathrm{CoO}_{2}(\mathrm{x}<0.5)$ during thermal runaway is given in Eqns 1-3 [4].

$\mathrm{Li}_{\mathrm{x}} \mathrm{CoO}_{2} \rightarrow \mathrm{Li}_{\mathrm{x}} \mathrm{Co}_{(1-\mathrm{y})} \mathrm{O}_{2-2 \mathrm{y}}+1 / 3 \mathrm{y} \mathrm{Co}_{3} \mathrm{O}_{4}+1 / 3 \mathrm{y} \mathrm{O} \mathrm{O}_{2}(\mathrm{~g})$

$\mathrm{Co}_{3} \mathrm{O}_{4} \rightarrow 3 \mathrm{CoO}+1 / 2 \mathrm{O}_{2}(\mathrm{~g})$ 
$\mathrm{CoO} \rightarrow \mathrm{Co}+1 / 2 \mathrm{O}_{2}(\mathrm{~g})$

An analogous process can take place in other metal-oxide cathode materials as a function of Li content, leading to capacity fade in the best case and catastrophic breakdown in the worst.

In this work, we look at the problem of oxidation of cathode anion sites from a quantum mechanical perspective, using a density functional theory (DFT) approach. We show that the shift from redox chemistry at the cation (metal) site to oxidation of the oxygen atoms as a function of state-of-charge can be predicted by examining the weight of the partial density of states PDOS at the Fermi energy. By correlating our calculations to experimental observations, we show that electronic instabilities driven by unstable oxygen can be predicted by noting when the oxygen PDOS becomes a majority. This provides and easy and relatively fast method of screening materials for a safe level of delithiation is therefore a useful tool in the design of new materials as Li ion battery cathodes.

\section{1}

\section{Background}

Oxidation of $\mathrm{O}^{2-}$ ions in a cathode compound can be understood from a quantum mechanical perspective by considering how electronic states are formed and occupied in the cathode material. During charging, electrons are withdrawn from the highest filled electronic state defined as the Fermi energy $\left(E_{F}\right)$. In a typical transition metal oxide cathode, this state is the anti-bonding state formed by hybridization of a cationic $3 d$ and an oxygen $2 p$ state, as shown in Figure 1 using a $\mathrm{Co}^{3+} / \mathrm{Co}^{4+}$ redox couple as an example. 


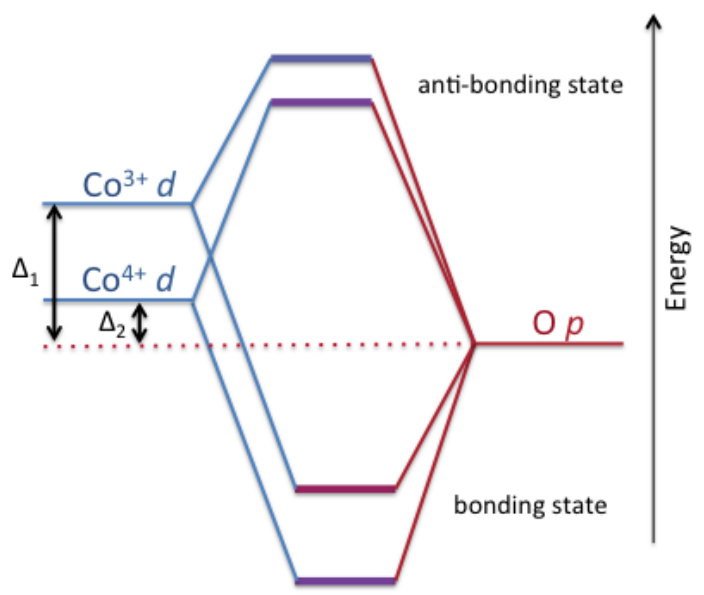

Figure 1: A schematic of the bonding/antibonding states of $\mathrm{Co}$ and $\mathrm{O}$ for two valencies of $\mathrm{Co}\left(\mathrm{Co}^{3+}\right.$ and $\left.\mathrm{Co}^{4+}\right)$. The strength of the splitting and hybridization depends on the distance between states as $1 / \Delta$. For $\mathrm{Co}^{3+}$, the distance $\Delta_{1}$ mixes relatively less oxygen into the anti-bonding states than the distance $\Delta_{2}$ for $\mathrm{Co}^{4+}$.

The admixture of oxygen into anti-bonding and cobalt into bonding states is determined by the strength of hybridization which is given analytically (to second order) as $t^{2} / \Delta$, where $\Delta$ is the energy distance between the $3 d$ and $2 p$ states and $t$ is the overlap of the orbitals. Both these quantities depend on the structure of the material and the oxidation and spin state of the cations [5]. Quite generally, though, it is clear that a lower energy $3 d$ state produces stronger hybridization and admixture via a smaller $\Delta$. Since the energy of a state depends on its electron occupancy, the valence of a $3 d$ ion is inversely related to its energy. Thus, a higher oxidation state in the metal $3 d$ cation corresponds to higher oxygen content in the anti-bonding state from which an electron will be withdrawn.

In a real material, the energy states as described above are broadened into energy bands, which can be calculated from first principles. Subsequently, the amount of oxygen and metal weight in the anti-bonding band at the Fermi energy can be calculated. In the case that the oxygen weight is comparable to the metal $3 d$ weight, any electrons withdrawn from this band, for instance during charging, will come substantially from around the real space oxygen site instead of the transition metal site. In other words, the expected redox reaction is violated in favor of oxidizing the oxygen, giving rise to possible formation of peroxide $\left(\mathrm{O}_{2}{ }^{2-}\right)$, and then $\mathrm{O}_{2}$ gas:

$$
\begin{aligned}
& 2 \mathrm{O}^{2-}=\mathrm{O}_{2}{ }^{2-}+2 \mathrm{e}^{-} \\
& \mathrm{O}_{2}{ }^{2-}=\mathrm{O}_{2}+2 \mathrm{e}^{-}
\end{aligned}
$$


The energy level of the oxygen bands, therefore, sets a limit on redox reactions. Goodenough showed that the voltage of a $3 d$ material is related to the height of the $3 d$ bands in the material and described the maximum voltage from a $3 d$ redox couple as being "pinned" to the level of the $\mathrm{O} 2 p$ bands [6]. From a different perspective, Huggins proposed that the operational potential of a battery is a suspect in its oxygen stability/safety, regardless of the materials chemical compositions and the crystal structures of the phases involved, by relating the Gibb’s free energy to the oxygen partial pressure and suggested that high voltages would inevitably lead to $\mathrm{O}_{2}$ formation [7].

We take the perspective that the voltage constraint, oxygen instability and "pinning" phenomena can be understood and even predicted by an accurate determination of the amount of oxygen mixed into the states at $\mathrm{E}_{\mathrm{F}}$. Simply put, we state that if the amount of $\mathrm{O}$ is equal to or greater than the amount of transition metal character in the band from which electrons are withdrawn, the system will be unstable toward oxidation of $\mathrm{O}^{2-}$ and oxygen gas release. We further propose that relatively simple first principles density functional theory (DFT) calculations can provide this accurate determination via a calculation of the partial density of states (PDOS). We calculate the metal/oxygen admixture for a selection of cathode materials with a broad range of structures, compositions, and voltages to gauge the oxygen instability. When possible, we make correspondence to available experimental data. This methodology is simple, effective, and requires many fewer calculations than would an evaluation of oxygen stability via full chemical reactions and materials decomposition, even at the simplest level as given in Equations 1-3.

\section{2}

\section{Materials}

We choose five materials for our calculations, the structures of which are shown in Figure 2. First we examine two extreme cases: $\mathrm{LiFePO}_{4}$, which is highly stable throughout the charge cycle, and $\mathrm{Li}_{2} \mathrm{CuO}_{2}$ which is highly unstable even at the beginning of the charge cycle. $\quad \mathrm{LiFePO}_{4}$ has the olivine structure both in the fully lithiated 
and fully delithiated states [8]. $\mathrm{Li}_{2} \mathrm{CuO}_{2}$ begins as the planar $\mathrm{Immm}$ structure, but transforms to the $C 2 / m$ structure (not shown) during cycling while simultaneously losing oxygen and experiencing severe capacity loss at each cycle [9]. Having used these two examples to bookend the limits of stability, we examine the practical battery material, $\mathrm{LiCoO}_{2}$ and its structural counterpart: $\mathrm{LiNiO}_{2}$. Both are isostructural layered materials of the $R \overline{3} m$ space group [10],[11]. $\mathrm{Li}_{\mathrm{x}} \mathrm{CoO}_{2}$ is a well-known and stable cathode material, as long as $\mathrm{x}>0.5$. However, as seen by Gupta and Manthiram [3], further delithiation can lead to oxygen instability in the following progressive oxidation reactions to peroxide in which the $O$ is in a -1 oxidation state (Eq. 4) and then $\mathrm{O}_{2}$ gas in which its oxidation state is effectively 0 (Eq. 5). Oxide coatings on $\mathrm{LiCoO}_{2}$ have been shown to suppress $\mathrm{O}_{2}$ evolution in Eq. 5 [12], a complex interaction with the electrolyte that is still under investigation.[13] $\mathrm{LiNiO}_{2}$ and its modified counterpart, $\mathrm{Li}\left[\mathrm{Ni}_{0.2} \mathrm{Li}_{0.2} \mathrm{Mn}_{0.6}\right] \mathrm{O}_{2}$, lose oxygen on their first charge cycle, causing a subsequent decrease in capacity $[14,15]$. In thermal analysis, $\mathrm{LiNiO}_{2}$ loses $\mathrm{O}_{2}$ at a lower temperature than $\mathrm{LiCoO}_{2}$, indicating that it is less stable $[2,16]$.

Finally, we investigate $\mathrm{Li}_{2} \mathrm{RuO}_{3}$ which is interesting because of its high capacity and its excellent conductivity deriving from a semi-metallic nature $[17,18]$. This compound, when doped with tin or manganese has recently been shown to derive additional capacity from formation of reversible peroxo- groups rather than $\mathrm{O}_{2}$ during charging [19]. $\mathrm{Li}_{2} \mathrm{RuO}_{3}$ has a $C 2 / c$ structure, which is a variation on the $R \overline{3} m$ structures, but with some of the metal sites instead occupied by Li.

a)
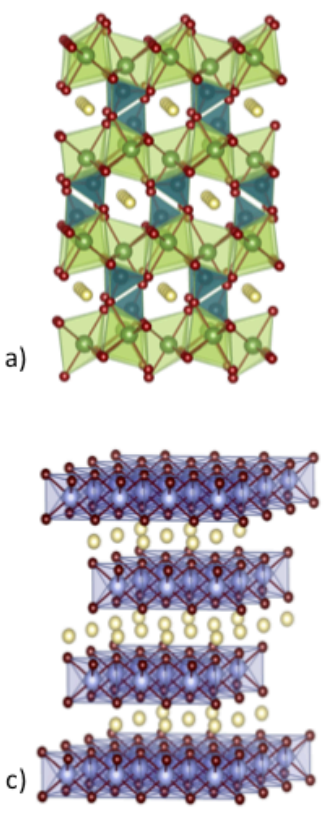

b)
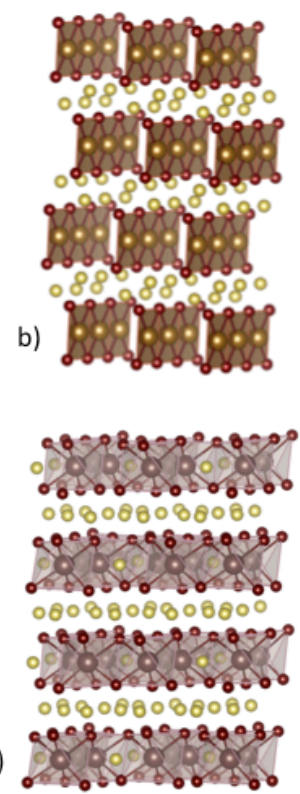

Figure 2: Crystal structures of (a) olivine, Pnmb $\mathrm{LiFePO}_{4}$, (b) $I m m m \mathrm{Li}_{2} \mathrm{CuO}_{2}$, (c) $R \overline{3} m \mathrm{LiCoO}_{2}$ and $\mathrm{LiNiO}_{2}$ and (d) $C 2 / c \mathrm{Li}_{2} \mathrm{RuO}_{3}$ 


\section{Computational Methods}

DFT has been widely advertised as a tool to study the electronic attributes and structural phases of battery materials $[20,21]$. The methodology is based on establishing the ground state charge density, and thereby the density of states, magnetic configuration, wave functions, etc., of a system with many atoms by solving multiple single particle Schrodinger-like equations with an effective potential (mean field) representing the net effect of the many-electron system. It has been shown that DFT can accurately reproduce measured structural parameters and open circuit voltages in a wide variety of Li ion battery cathode materials [22].

Within DFT, there is a necessary approximation to a part of the energy potential, called the exchange-correlation potential. Mostly commonly used are the standard local density approximation and generalized gradient approximations (LDA and GGA) [23, 24]. These have been shown to be very accurate for systems without highly localized electrons. However, the majority of redox couples in $\mathrm{Li}^{+}$cathode materials come from localized $3 d$ states for which the LDA/GGA approximations, while still providing accurate structural information, can fail badly at the electronic level. A very common remedy for the LDA/GGA shortcomings is the LDA+U theory, in which a Hubbard-like "U" term is added to the potential [25]. LDA+U theory can be effective in obtaining the correct electronic ground state and is computationally efficient, but requires a separate choice of "U" for each element within a material and each set of valence orbitals of that element. Furthermore, the choices for " $U$ " are not universal; values vary across structural types and valencies. While the vast majority of orbitals require no "U" correction at all, an inaccurate choice for those that do can result in an incorrect sequence of redox couples in mixed cationic materials and/or erroneous voltage profiles. Most importantly for our purposes, it will not correctly reproduce the hybridization level in the anti-bonding level at the Fermi energy. 
For this work, we choose a functional that treats all electronic states on the same footing rather than applying a correction to a selected subset. This eliminates the both necessity of choosing individual parameters for each element/orbital as well as of defining the orbitals themselves from density. In specific, we use the HSE06 functional [26] as implemented in the Vienna Ab-initio Simulation Program (VASP) [27]. While using this functional has the unfortunate effect of increasing the computational expense by an order of magnitude, it has been shown to correctly reproduce the position of $d$ bands relative to oxygen bands [28] and to correctly establish voltages in metal oxide cathode materials [29].

We fully relax the lattice constants and ionic positions of all constituent atoms at each examined $\mathrm{Li}$ concentration until they reach their lowest energy state. We calculated systems with multiple Li orderings and found that different ordering produce small changes in energy and even smaller changes in the densities of states. We chose the lowest calculated energy configuration for each final presented set of data. All relaxations are performed with a 6x6x4 grid of k-points followed by a final self-consistent calculation with 12x12x8 kpoints in the lowest energy cell. For simplicity the spin majority and spin minority states have been added together and plotted on the same axis.

\section{0}

\section{Results: PDOS Calculations}

\section{1}

\section{Stable and unstable endpoints}

We begin with a demonstration of two contrasting compounds: oxygen stable $\mathrm{Li}_{\mathrm{x}} \mathrm{FePO}_{4}$, and oxygen unstable $\mathrm{Li}_{\mathrm{x}} \mathrm{CuO}_{2}$. The PDOS for these compounds are presented in Figure 3. Each $\mathrm{Li}^{+}$ion withdrawn during charging shifts one electron's worth of states below $E_{F}$ to empty electron states above $E_{F}$, as indicated by arrows in the Figure. For $\mathrm{Li}_{\mathrm{x}} \mathrm{FePO}_{4}$, the withdrawal of one electron per formula unit represents full charge. The electron state that moves across $\mathrm{E}_{\mathrm{F}}$ in this system is dominated by the Fe contribution with a tiny admixture of oxygen. By subtracting the charge densities calculated before and after electron withdrawal, we can image the hole left behind in the material. In $\mathrm{FePO}_{4}$, the cloud of charge missing from the system is localized around a Fe site with 
very small remnants near oxygen. This indicates a metal valence change and the typical redox chemistry associated with the charge/discharge process in stable cathodes, leaving the oxygen in its preferred 2-valency. In contrast, the states that cross $\mathrm{E}_{\mathrm{F}}$ upon withdrawal of one electron in $\mathrm{Li}_{\mathrm{x}} \mathrm{CuO}_{2}$ are dominated by oxygen, with only minority $\mathrm{Cu}$ content. As can be seen in the charge density plot, the missing charge is delocalized and has been pulled heavily from around oxygen atoms, leaving the compound susceptible to peroxide formation.
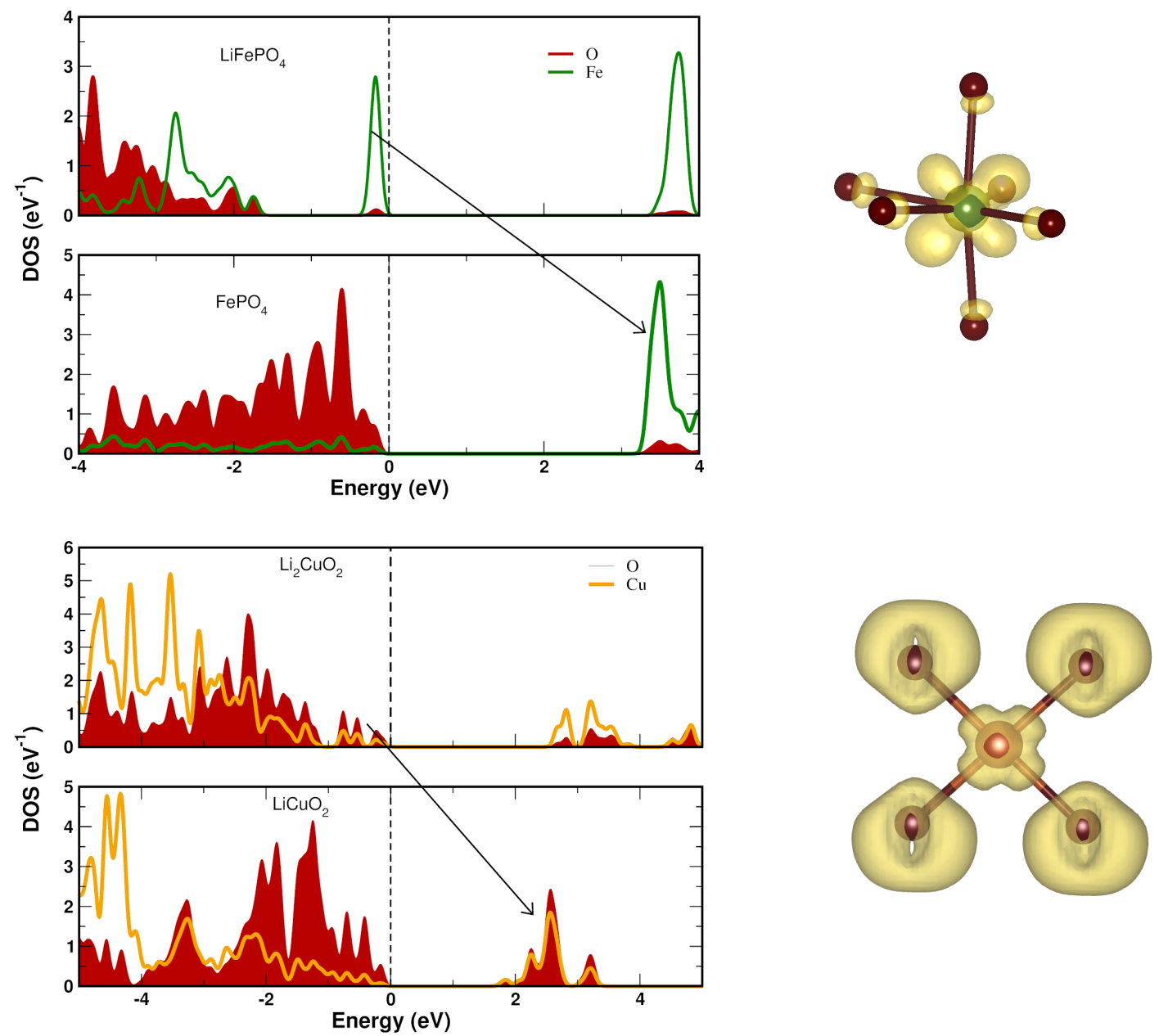

Figure 3: Partial density of states for $\mathrm{M}$ and $\mathrm{O}$ in $\mathrm{Li}_{\mathrm{x}} \mathrm{FePO}_{4} / \mathrm{Li}_{2} \mathrm{CuO}_{2}$ at full discharge (top) and full/partial charge (bottom). Filled states are to the left of the Fermi energy (set to zero) and empty states to the right. A close-up of the hole states left behind after withdrawal of an electron are shown adjacent to each DOS plot. The removed charge is clearly centered around $\mathrm{Fe}$ for $\mathrm{LiFePO}_{4}$, but comes heavily from oxygen in $\mathrm{Li}_{2} \mathrm{CuO}_{2}$. 
There is yet one further $\mathrm{Li}^{+}$ion available to be withdrawn, once the Cu-based compound has reached $\mathrm{LiCuO}_{2}$. Were it to be removed, the associated electron would come from the states below $\mathrm{E}_{\mathrm{F}}$ in the bottom panel. These are almost entirely composed of oxygen and removing charge would even further destabilize the oxygen toward peroxide formation and finally $\mathrm{O}_{2}(\mathrm{~g})$.

The results of the PDOS calculations in these materials can be correlated to their cycling properties. $\mathrm{Li}_{\mathrm{x}} \mathrm{FePO}_{4}$ is well known to be stable and repeatedly cycleable across the entire range of $x$, with almost no loss of oxygen [30]. $\mathrm{Li}_{x} \mathrm{CuO}_{2}$, on the other hand, exhibits immediate and dramatic capacity loss when cycled [31]. While a standard redox reaction would proceed reversibly according to Eq. 6, when $\mathrm{O}^{2-}$ is oxidized, the reaction is no longer fully reversible, peroxide is formed along with by-product phase $\mathrm{CuO}$, and capacity is lost at each step, according to Eqs. 7,8.

$$
\begin{aligned}
& \mathrm{Li}_{2} \mathrm{CuO}_{2} \leftrightarrow \mathrm{Li}_{2-\mathrm{x}} \mathrm{CuO}_{2}+\mathrm{xLi}^{+}+\mathrm{e}^{-} \\
& \mathrm{Li}_{2-\mathrm{x}-\mathrm{y}} \mathrm{CuO}_{2} \leftrightarrow \mathrm{CuO}+\mathrm{yLi}^{+}+1 / 2 \mathrm{O}_{2}+\mathrm{e}^{-}
\end{aligned}
$$

The loss of $\mathrm{O}_{2}$ gas has been inferred by the formation of $\mathrm{CuO}$ phases forming in conjunction with irreversible capacity loss during the cycling of $\mathrm{Li}_{2} \mathrm{CuO}_{2}[31]$.

\section{2}

\section{Layered transition metal cathodes}

Having shown that the PDOS correlates to oxygen stability/instability, at least in very clear-cut cases, we examine the more complex cases of $\mathrm{LiCoO}_{2}$ and $\mathrm{LiNiO}_{2}$, two isostructural compounds which are neither fully stable nor fully unstable. These have been calculated before by Laubach, et al. [32]. By their calculations, the Co PDOS always was above the O PDOS at the Fermi level, although there was extensive hybridization between the $\mathrm{Co} 3 \mathrm{~d}$ and $\mathrm{O} 2 \mathrm{p}$ states at $\mathrm{x}<0.5$ of $\mathrm{O}^{2-}$ anions. By comparison, $\mathrm{LiNiO}_{2}$ showed the reverse, with the O PDOS always higher than the Ni PDOS, although the authors were not as confident with this computational result. The source of their hesitation was their use of $\mathrm{DFT}+\mathrm{U}$, which as mentioned previously, may skew the 
energy order of the Ni and $\mathrm{O}$ states and which we avoid with our use of HSE06. Xiao et al. [33], also using DFT+U, calculated the PDOS of $\mathrm{Li}_{2} \mathrm{MO}_{2}$ with $\mathrm{M}=\mathrm{Ti}$, Mn,Co which has the same structure as $\mathrm{LiCoO}_{2}$ but with 1/3 of the transition metal species replaced by Li. This work emphasized the connection between the PDOS and oxygen stability, as we do here, and provides an additional example of the utility of the method.

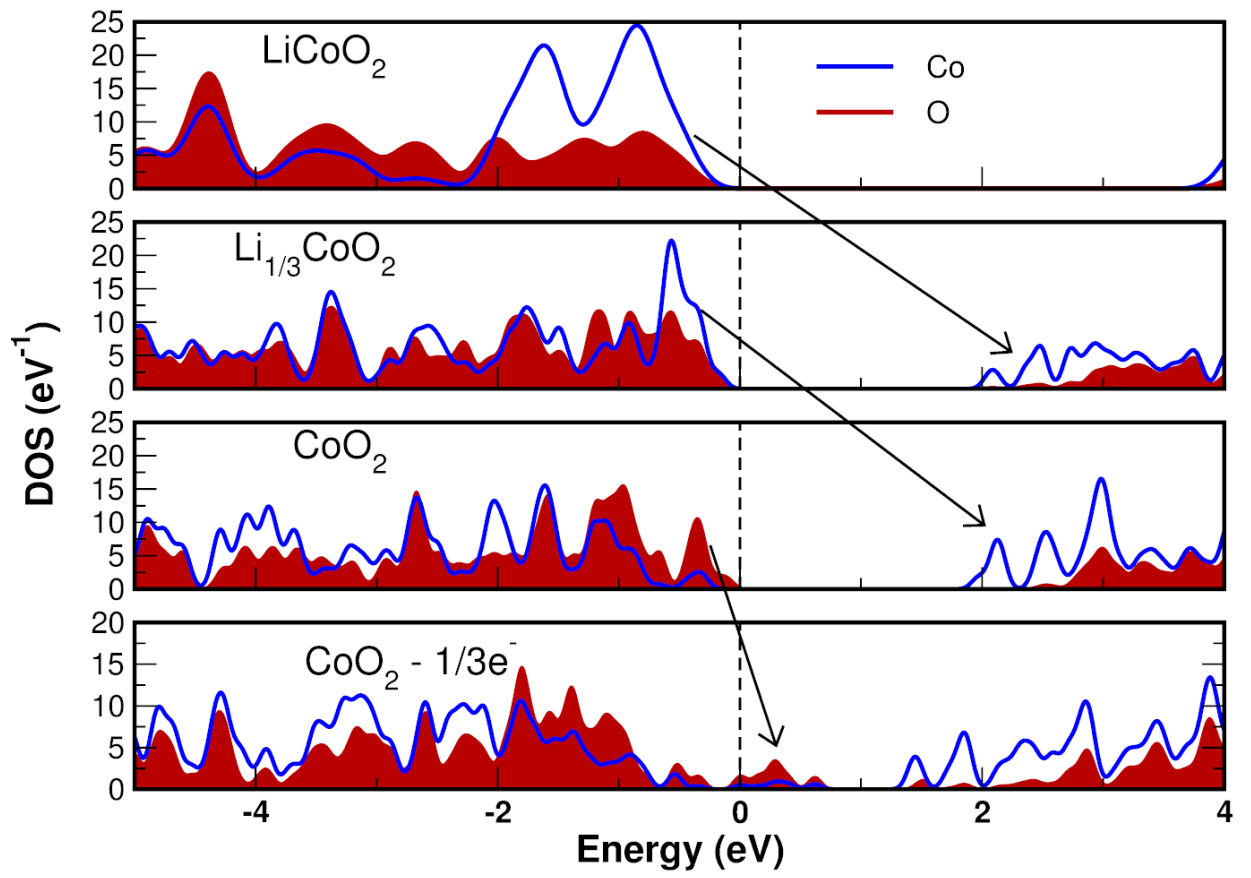

Figure 4: PDOS of successive stages of delithation of $\mathrm{Li}_{\mathrm{x}} \mathrm{CoO}_{2}$ with all energies shifted so that $\mathrm{E}_{\mathrm{F}}$ is at zero. Arrows point from filled states at one stage to emptied states at the next stage. The lower panel shows a predicted PDOS for a compound in which $1 / 6$ further electrons are withdrawn, even after all the $\mathrm{Li}$ is gone.

Figure 4 shows $\mathrm{Li}_{x} \mathrm{CoO}_{2}$ for $\mathrm{x}=1, \mathrm{x}=0.33$, and $\mathrm{x}=0$ in the top three panels. At full lithiation (top panel), the states just below $E_{F}$ have substantial oxygen character, but are majority Co. When 1/3 of the Li is removed, electrons pulled from these states are shifted above $\mathrm{E}_{\mathrm{F}}$ (second panel), where it can be seen that their character is somewhat reorganized, but remains mainly Co-like. The new states located at $\mathrm{E}_{\mathrm{F}}$ have an increased oxygen weight (compared to the discharged compound). When electrons are removed from these states to achieve the fully delithiated compound (third panel), a similar process occurs: the Co vs. O composition appears somewhat reorganized but Co character dominates, indicating that $\mathrm{Li}^{+}$and $\mathrm{e}^{-}$withdrawal should be chemically stable for the full range of Li content. At about $3 \mathrm{eV}$ above $\mathrm{E}_{\mathrm{F}}$, there are empty states with a substantial, though not 
dominant, amount of oxygen character. This means that charge has been removed from around the oxygen site, but is not conclusive in terms of whether or not a peroxide instability is incipient. It is certainly clear that the electron states below $\mathrm{E}_{\mathrm{F}}$ increase in oxygen content as $\mathrm{Li}$ is removed.

In the final panel of Figure 4, we use a computational technique to remove a further electron from the $\mathrm{LiCoO}_{2}$ system, despite there being no Li left. Specifically, we reduce the number of electrons in the system and charge compensate by using a uniform negative background charge that integrates to the exact number by which the electrons were reduced.

In this case, it is clear that the removed electrons have dominant oxygen character, indicating oxidation of the $\mathrm{O}^{2-}$ ions. Although experimental removal of electrons in precisely this manner is impossible, our calculated spectrum demonstrates that the $\mathrm{Co}^{3+} / \mathrm{Co}^{4+}$ redox couple is unavailable in this compound and attempts to access it would likely result in peroxide formation. A possible physical realization of this circumstance is a $\mathrm{Li}_{\mathrm{x}} \mathrm{CoO}_{2}$ compound with Co vacancies or with Co over-coordinated to O, such as might occur at surfaces in nanoscale materials, in systems with phase impurities such as $\mathrm{Co}_{3} \mathrm{O}_{4}$, or in mixed cationic systems with $\mathrm{Co}^{3+}$ as a component. Indeed, the observed instability of $\mathrm{LiCoO}_{2}$ increases if the $\mathrm{LiCoO}_{2}$ particles are nanoscale [34].

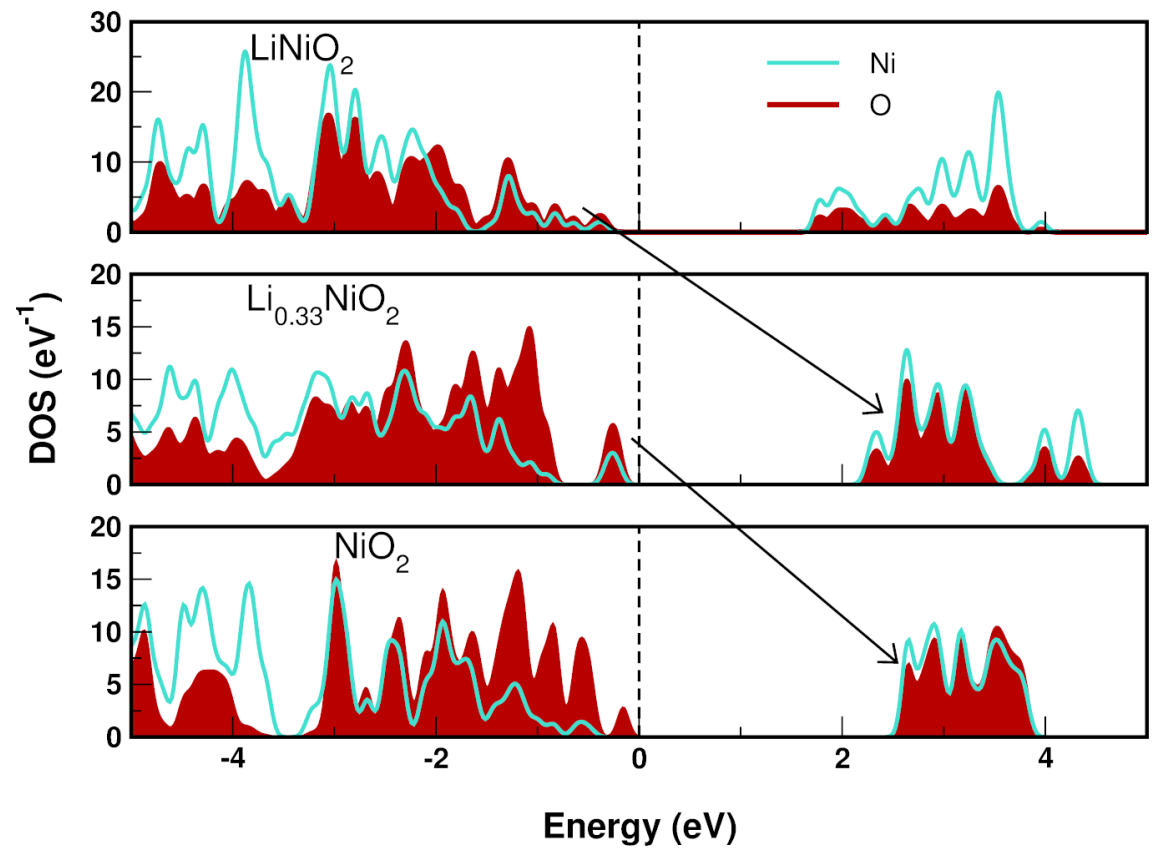

Figure 5: The PDOS of $\mathrm{Li}_{x} \mathrm{NiO}_{2}$ at various $\mathrm{Li}$ content. The Fermi energy has been shifted to zero. Arrows point from filled states at one stage to emptied states at the next stage. 
Figure 5 shows the PDOS of $\mathrm{Li}_{x} \mathrm{NiO}_{2}$, which is isostructural to $\mathrm{LiCoO}_{2}$ (at least at full lithiation) but has one additional electron in the system. Looking at the top panel of Figure 5, compared to the top panel of Figure 4, one can immediately see that the Ni-based compound has significantly more oxygen in the states just below $\mathrm{E}_{\mathrm{F}}$. In fact, the oxygen is dominant for $\mathrm{LiNiO}_{2}$ at the top of the filled spectrum. When $\mathrm{Li}^{+}$ions and electrons are removed to form $\mathrm{Li}_{0.33} \mathrm{NiO}_{2}$, one can see that the $\mathrm{Ni} / \mathrm{O}$ contributions are reorganized somewhat (as was seen in the Co compound), such that the emptied states above $E_{F}$ are now Ni dominated, though just barely. There is heavy oxygen character in these states, indicating that $\mathrm{O}^{2-}$ is likely partially oxidized during charging.

Thomas, et al. [11], claimed that in $\mathrm{Li}_{\mathrm{x}} \mathrm{NiO}_{2}$, lithium could not be extracted for $\mathrm{x}<0.4\left(<4.25 \mathrm{~V}\right.$ vs $\left.\mathrm{Li}^{+} / \mathrm{Li}^{0}\right)$ because the lack of interaction of between the layers brought on by loss of mediating $\mathrm{Li}^{+}$ions would necessitate an unfavorable Ni valency combined with ionic migration to stave off structural reorganization. Our calculations show that slightly before this point, a structural or chemical reorganization will be brought about by oxidation of the $\mathrm{O}^{2-}$ ions. This can surely be related to the unfavorable Ni valency of Thomas et al., but our perspective differs in that it is an undesirable oxygen valency, precipitated by the high Ni valency, which brings the d-bands very low in energy and drives the instability. The difference in the metal oxide stability and energetic barriers to high $\mathrm{Li}$ extraction between $\mathrm{LiCoO}_{2}$ and $\mathrm{LiNiO}_{2}$ are borne out by experimental results, which confirm that $\mathrm{LiNiO}_{2}$ loses $\mathrm{O}_{2}$ at a much lower temperatures than $\mathrm{LiCoO}_{2}$ during thermal analysis measurements [16]. $\mathrm{LiNiO}_{2}$ is also known to lose significant oxygen and thus capacity on its first charge cycle. The good correspondence between the practical measurements of $\mathrm{O}$ loss and the calculated oxygen admixture into the relevant electronic states provides strong evidence that the PDOS calculated with DFT (using an accurate functional) embodies the global changes in the $\mathrm{M}$ and $\mathrm{O}$ electronic states of the materials, and can be used as a predictive tool for oxygen stability.

\section{3}

\section{Ruthenate cathodes}

Finally, we present PDOS calculations to investigate the oxygen stability of $\mathrm{Li}_{2} \mathrm{RuO}_{3}$, variations of which are now being suggested as high capacity materials through electron contributions from their 
oxygen ions via $\mathrm{O}$ anion oxidation to peroxide (Eq 5). The top panel of Fig. 8 shows the fully discharged system with Ru-dominated states just below $\mathrm{E}_{\mathrm{F}}$. Upon removal of one $\mathrm{Li}^{+}$ion and one electron (middle panel), these states move above $\mathrm{E}_{\mathrm{F}}$ and remain mainly Ru-like, indicating that this step accesses the $\mathrm{Ru}^{4+} / \mathrm{Ru}^{5+}$ redox couple and should be stable and fully reversible. The next states to be accessed at $\mathrm{E}_{\mathrm{F}}$ have significantly heavier oxygen character, though Ru is still barely the majority. The final panel shows this next step, which is ostensibly accessing the $\mathrm{Ru}^{5+} / \mathrm{Ru}^{6+}$ redox couple. However, in the emptied states above $\mathrm{E}_{\mathrm{F}}$, $\mathrm{Ru}$ character and $\mathrm{O}$ character are approximately equal, indicating a propensity toward oxidation of the $\mathrm{O}^{2-}$ ions to $\mathrm{O}_{2}{ }^{2-}$ and eventual formation of $\mathrm{O}_{2}$. The spectra is reminiscent of delithiated $\mathrm{LiNiO}_{2}$ in which heavy oxygen character exists in the emptied states, but the transition metal bands are still perhaps slightly above the oxygen bands. Looking at the spectrum now below $\mathrm{E}_{\mathrm{F}}$ in the bottom panel, it is clear that the states are mainly of oxygen-like character. In other words, an attempt to access the $\mathrm{Ru}^{6+} / \mathrm{Ru}^{7+}$ couple will surely result instead in a change to the oxygen valency. Recently published literature indicates that in systems where $\mathrm{Ru}$ is partially replaced by electrochemically inactive ions, such as $\mathrm{Mn}^{4+}$ or $\mathrm{Sn}^{4+}$ there is peroxide $\left(\mathrm{O}_{2}{ }^{2-}\right)$ formation at high levels of delithiation [35]. This is in perfect agreement with our calculations since $\mathrm{Mn}^{4+}$ or $\mathrm{Sn}^{4+}$ would force the system to access the $\mathrm{Ru}^{6+} / \mathrm{Ru}^{7+}$ couple where oxygen states dominate. In Tarascon's work, ${ }^{[19],[35]}$ the peroxide formation is a reversible source of capacity via Eq. 4 and does not lead to an overall materials instability via irreversible $\mathrm{O}_{2}$ loss (Eq 5). This seems to be a unusual state of affairs in cathode materials, as $\mathrm{O}_{2}$ loss has previously only been prevented by coating lithium metal oxides with inert metal oxides [12, 13]. It is not clear why peroxide formation provides extra (and reversible) capacity rather than an instability in the case of the doped $\mathrm{Li}_{2} \mathrm{RuO}_{3}$ compounds, but this oxidation step does not progress to $\mathrm{O}_{2}$ (g) loss and overall battery instability as it does in others $\left(\mathrm{LiCoO}_{2}, \mathrm{LiNiO}_{2}\right.$, etc.). Unfortunately, $\mathrm{Li}_{2} \mathrm{RuO}_{3}$ is not a heavily studied material, and there are no reports of $\mathrm{Li}_{2} \mathrm{RuO}_{3}$ oxygen stability, beyond the work of Sarkar, et al. which infers $\mathrm{O}_{2}$ loss on the first charge cycle [36]. Our 


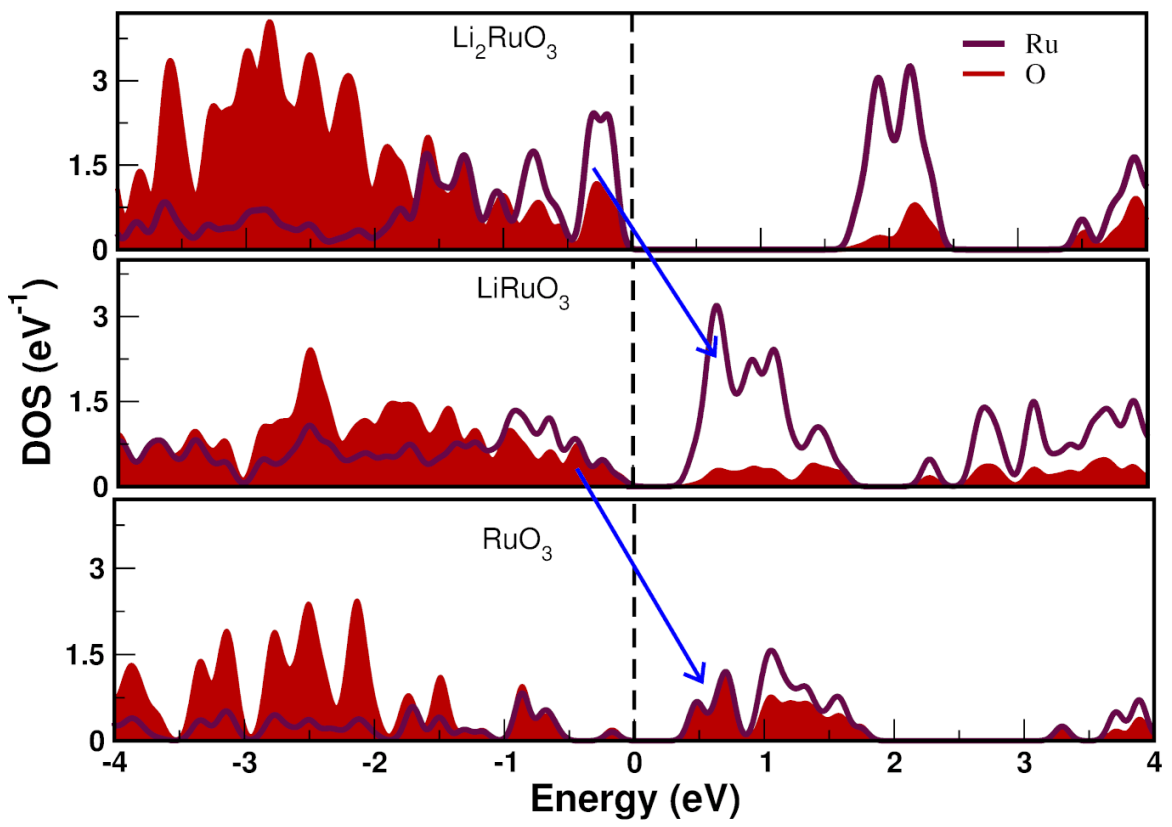

Figure 6: The PDOS of $\mathrm{Li}_{x} \mathrm{RuO}_{3}$ at various Li content. The Fermi energy has been shifted to zero. Arrows point from filled states at one stage to emptied states at the next stage.

prediction method, therefore, is shown to accurately gauge the instability of the oxygen toward peroxide formation, but is not able to predict why the peroxide remains stable in the material.

There are other indications in the literature of other materials with anomalously high $\mathrm{Li}^{+}$capacity, which may be attributable to peroxide formation such as high surface area $\mathrm{V}_{2} \mathrm{O}_{5}$ [37]. Capacities on the order of $4 \mathrm{Li}^{+}$per $\mathrm{V}$ cation are reported in $\mathrm{V}_{2} \mathrm{O}_{5}$ aerogels, suggesting an unlikely $\mathrm{V}^{5+} / \mathrm{V}^{1+}$ redox couple. It was postulated that vanadium vacancies in the high surface area structure could serve as additional sites for charge balance during $\mathrm{Li}^{+}$insertion, without a change in $\mathrm{V}$ valency [38]. However, in the delithiated material, since no further $\mathrm{d}$ electrons (beyond $\mathrm{V}^{5+}$ ) are available, the likely result of such vacancies is partial oxidation of oxygen sites. Note that $\mathrm{V}_{2} \mathrm{O}_{5}$ aerogel are also highly unstable to cycling, consistent with $\mathrm{O}_{2}$ loss through the reduction of $\mathrm{O}$ sites.

At the simple level of PDOS only, our calculations cannot determine the final state that the $\mathrm{O}^{2-}$ ions take during 
excessive delithiation in materials such as $\mathrm{Li}_{2} \mathrm{RuO}_{3}$ or high surface area $\mathrm{V}_{2} \mathrm{O}_{5}$, but can only predict when the first step toward $\mathrm{O}_{2}$ (g) release, i.e. peroxide formation, is likely in the bulk material. Further experimental and computational studies to illuminate the underlying mechanisms that stabilize the oxidized oxygen states are highly desirable. The role of the surface in the peroxide formation is also another key area for research.

\section{0}

\section{Discussion}

Our DFT calculations for the PDOS of the M and $\mathrm{O}$ character of delithiated metal oxides match well to experimental observations of their propensity to lose, or evolve, $\mathrm{O}_{2}$ upon delithiation or charging. The electrochemical and structural attributes of the five materials studied in this report are summarized in Table I.

Table 1: Attributes of the four Li-ion battery cathode materials compared in this article, presenting a range of potentials, stability/cyclability, and structures.

\begin{tabular}{|c|c|c|c|c|c|c|c|}
\hline $\begin{array}{l}\text { Discharged } \\
\text { Cathode }\end{array}$ & $\begin{array}{l}\text { Charged } \\
\text { Cathode }\end{array}$ & Structure & $\begin{array}{l}\text { Redox plateau vs } \\
\mathrm{Li}^{+} / \mathrm{Li}^{0}\end{array}$ & $\begin{array}{l}\text { Capacit } \\
\mathrm{y} \\
\mathrm{mAh} / \mathrm{g}\end{array}$ & $\begin{array}{l}\text { Operational } \\
\text { voltage } \\
\text { range (V) }\end{array}$ & $\begin{array}{l}\text { Voltage for } \\
\mathrm{O}_{2} \\
\text { evolution }\end{array}$ & REF \\
\hline $\mathrm{LiCoO}_{2}$ & $\mathrm{Li}_{0.5} \mathrm{CoO}_{2}$ & $R \overline{3} m$ & $3.8 \mathrm{~V} \mathrm{Co}^{3+} / \mathrm{Co}^{4+}$ & 140 & $2.7-4.2 \mathrm{~V}$ & $>4.6 \mathrm{~V}$ & $\begin{array}{l}{[4],[10],} \\
{[16]}\end{array}$ \\
\hline $\mathrm{LiNiO}_{2}$ & $\mathrm{Li}_{0.5} \mathrm{NiO}_{2}$ & $R \overline{3} m$ & $3.4 \mathrm{~V} \mathrm{Ni}^{3+} / \mathrm{Ni}^{4+}$ & 140 & $2.5-3.9 \mathrm{~V}$ & $4.2 \mathrm{~V}$ & $\begin{array}{l}{[4],[11],} \\
{[16]}\end{array}$ \\
\hline $\mathrm{Li}_{2} \mathrm{RuO}_{3}$ & $\mathrm{LiRuO}_{3}$ & $C 2 / C$ & $3.65 \mathrm{~V} \mathrm{Ru}^{4+} / \mathrm{Ru}^{5+}$ & 160 & $3.0-4.0 \mathrm{~V}$ & $\begin{array}{l}\text { unknown } \\
\text { [19] }\end{array}$ & $\begin{array}{l}{[18,19,} \\
35]\end{array}$ \\
\hline $\mathrm{Li}_{2} \mathrm{CuO}_{2}$ & $\mathrm{LiCuO}_{2}$ & Immm to $C 2 / m$ & $\begin{array}{l}3.0 \mathrm{~V} \mathrm{Cu}^{2+} / \mathrm{Cu}^{3+} \\
2.5 \mathrm{~V} \mathrm{Cu}^{2+} / \mathrm{Cu}^{1+}\end{array}$ & 180 & $1.5-3.7 \mathrm{~V}$ & $>2.0 \mathrm{~V}$ & $\begin{array}{ll}9, & 31, \\
39] & \end{array}$ \\
\hline $\mathrm{LiFePO}_{4}$ & $\mathrm{Li}_{0.5} \mathrm{FePO}_{4}$ & Pnmb & $3.4 \mathrm{~V} \mathrm{Fe}^{3+} / \mathrm{Fe}^{4+}$ & 110 & $2.5-4.0 \mathrm{~V}$ & N/A & {$[8,30]$} \\
\hline
\end{tabular}


The propensity of the materials to evolve oxygen during delithiation is shown in Figure 7. The area where the material is stable to cycling is in green, and its oxygen evolution region is in red, except for $\mathrm{Li}_{2} \mathrm{RuO}_{3}$ where the higher potentials are denoted in purple due to the uncertainly about whether the material loses $\mathrm{O}_{2}$ or forms a peroxide ion during charging, which can be reversed to oxygen anions during discharge. Interestingly, from Fig. 7, there appears to be no clear correlation to the operational potential of the material and its stability. The propensity of the material to evolve oxygen appears to be determined by a range of interdependent factors, including the valency of the electrochemically active ion, the material's crystal structure, and the local oxygen coordination, with no direct correlation to its $\mathrm{E}^{0}$ value, e.g. the $\Delta \mathrm{G}$ value of its reaction for $\mathrm{Li}+$ insertion (for example, Eq. 6). For $\mathrm{LiCoO}_{2}, \mathrm{LiNiO}_{2}$ and $\mathrm{Li}_{2} \mathrm{CuO}_{2}$, in fact, the propensity for $\mathrm{O}_{2}$ loss follows the reverse trend, and increases as the $\mathrm{E}^{0}$ value decreases, contradicting the proposal that high voltage cathode materials per se are less safe [7]. In the case of the $\mathrm{LiFePO}_{4}$, the $\mathrm{O}$ states themselves are lowered via strong bonding with the $\mathrm{P}$ anions, such that the electronic states always maintain a heavy $\mathrm{M}$ character, which is the so-called polyanionic inductive effect [40]. In the $3 d$ transition metal compounds with $\mathrm{Co}, \mathrm{Ni}$, and $\mathrm{Cu}$, the $3 d$ bands move down in energy as a function of increasing nuclear charge. $\mathrm{LiCoO}_{2}$ has little oxygen character at $\mathrm{E}_{\mathrm{F}}$ at each state of delithiation. $\mathrm{Li}_{x} \mathrm{NiO}_{2}$ starts to evolve $\mathrm{O}_{2}$ at $\mathrm{x}<0.4$ when the $\mathrm{Ni}^{4+}$ cation bands hybridize so strongly with oxygen

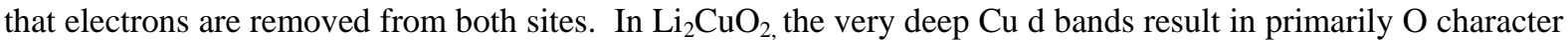
at $\mathrm{E}_{\mathrm{F}}$ even when fully lithiated. $\mathrm{In}_{\mathrm{Li}_{2}} \mathrm{RuO}_{3}$, the $\mathrm{O}^{2-}$ ions do not become oxidized until the valency is higher than $\mathrm{Ru}^{5+}$. This unusual stability owes to the fact that $4 d$ states are more weakly bound to the nucleus than $3 d$ states and therefore are located further from the oxygen states. 


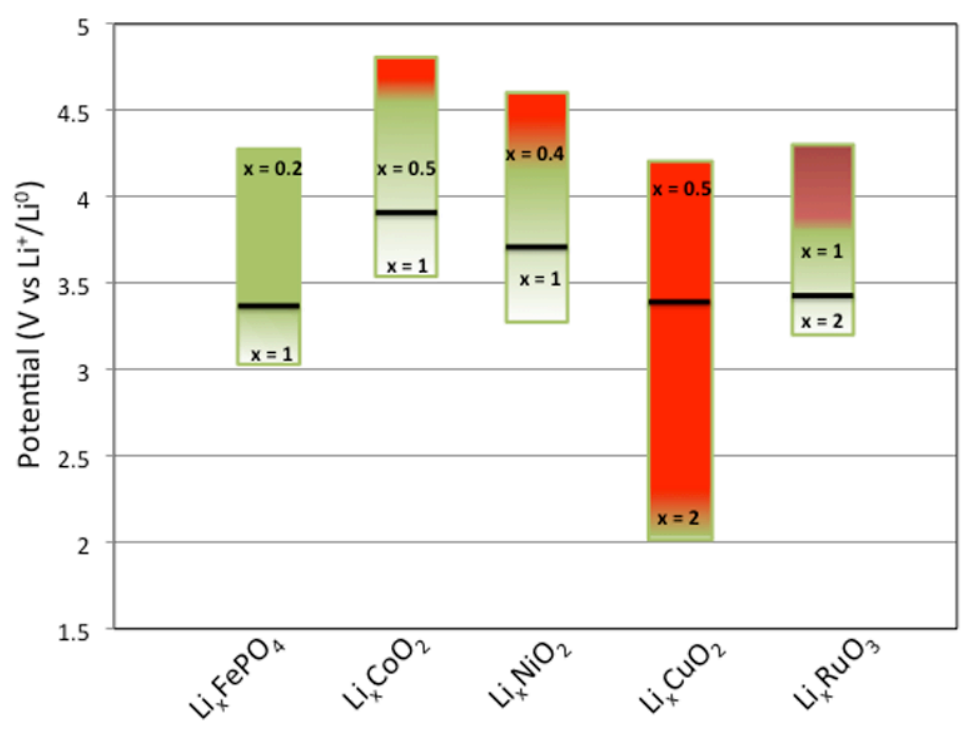

Figure 7: Schematic of voltage range for Li-ion battery cathodes vs variation in Li content (x). The nominal charge voltage of the material, or $\mathrm{E}^{0}$, is denoted with a black line. The voltage region in which the materials evolve $\mathrm{O}_{2}$ is shown in red, with the exception of $\mathrm{Li}_{2} \mathrm{RuO}_{3}$, which is thought to instead reversibly form stable peroxyl species on its surface [35].

Therefore, each material has a unique set of circumstances that govern its electronic character, and how it is oxidized during charging. Whereas it would be attractive to have a "one size fits all" theory for $\mathrm{O}_{2}$ evolution from lithium ion battery cathodes, it appears that each material must be studied individually, with consideration of its metal constituents, crystal structure, local coordination and orbital character. All of these factors can be taken into account simultaneously and accurately within DFT. Whether it eventually leads to actual $\mathrm{O}_{2}$ evolution, oxygen instability at the electrolyte, or a reversible structural or chemical change, oxidation of $\mathrm{O}^{2-}$ can be fundamentally understood and predicted through a relatively simple calculation of the PDOS of the cathode material.

\section{0}

\section{Conclusions}

Using DFT with the HSE06 functional that applies a correction to the exchange potential for both transition metal and oxygen orbitals, we are able to correlate the propensity of material to evolve $\mathrm{O}_{2}$ during 
charging or delithiation to its oxygen character in PDOS calculations. $\mathrm{O}_{2}$ loss appears to be strongly correlated to the underlying electronic structure of the material, and not its reaction potential toward $\mathrm{Li}^{+}$. When the presence of oxygen character in the states from which electrons are withdrawn during the charge cycle is greater than the metal character, the $\mathrm{O}$ anions in the material are destabilized. Oxidation of the oxygen anions causes instability and/or peroxide formation, and ultimately the propensity of a material to lose $\mathrm{O}_{2}$. Oxidation of the $\mathrm{O}^{2-}$ ions can also lead to capacity fade, structural phase transitions or even lead to thermal runaway when a reaction with the electrolyte occurs. While our calculations cannot predict the eventual electrochemical consequences of $\mathrm{O}^{2-}$ oxidation, they can serve as a predictor of possible instabilities. As researchers probe new materials, both experimentally and by computational discovery, these relatively straightforward PDOS measurements, if done accurately, are a useful tool for screening the electronic stability range as a function of state of charge.

\section{Acknowledgements}

We are grateful to the Office of Naval Research for support of this research through NRL's Advance Research Initiative on Metal Oxides and the Laboratory’s Basic Research Program.

\section{Notes and references}

\section{U. S. Naval Research Laboratory, Washington, DC 20375 USA}

[1] C.T. Love, M.B.V. Virji, R.E. Rocheleau, K.E. Swider-Lyons, J. Power Sources 266 (2014) 512.

[2] J.R. Dahn, E.W. Fuller, M. Obrovac, U. Vonsacken, Solid State Ion. 69 (1994) (3-4) 265.

[3] R. Gupta, A. Manthiram, Journal of Solid State Chemistry 121 (1996) (2) 483.

[4] Q.S. Wang, P. Ping, X.J. Zhao, G.Q. Chu, J.H. Sun, C.H. Chen, J. Power Sources 208 (2012) 210.

[5] J.B. Goodenough, Progress in Solid State Chemistry 5 (1971) 145.

[6] J.B. Goodenough, Y. Kim, Chem. Mat. 22 (2010) (3) 587.

[7] R.A. Huggins, J. Electrochem. Soc. 160 (2013) (5) A3001.

[8] K. Zaghib, A. Guerfi, P. Hovington, A. Vijh, M. Trudeau, A. Mauger, J.B. Goodenough, C.M. Julien, J. Power Sources 232 (2013) 357. 
[9] H. Arai, S. Okada, Y. Sakurai, J. Yamaki, Solid State Ion. 106 (1998) (1-2) 45.

[10] K. Mizushima, P.C. Jones, P.J. Wiseman, J.B. Goodenough, Mater. Res. Bull. 15 (1980) (6) 783.

[11] M.G.S.R. Thomas, W.I.F. David, J.B. Goodenough, P. Groves, Mater. Res. Bull. 20 (1985) (10) 1137.

[12] Y.J. Kim, J.P. Cho, T.J. Kim, B. Park, J. Electrochem. Soc. 150 (2003) (12) A1723.

[13] J. Hong, H.D. Lim, M. Lee, S.W. Kim, H. Kim, S.T. Oh, G.C. Chung, K. Kang, Chem. Mat. 24 (2012) (14) 2692.

[14] A.R. Armstrong, M. Holzapfel, P. Novak, C.S. Johnson, S.H. Kang, M.M. Thackeray, P.G. Bruce, Journal of the American Chemical Society 128 (2006) (26) 8694.

[15] T.A. Arinkumar, Y. Wu, A. Manthiram, Chem. Mat. 19 (2007) (12) 3067.

[16] D.D. MacNeil, Z.H. Lu, Z.H. Chen, J.R. Dahn, J. Power Sources 108 (2002) (1-2) 8.

[17] H. Kobayashi, R. Kanno, Y. Kawamoto, M. Tabuchi, O. Nakamura, M. Takano, Solid State Ion. 82

(1995) (1-2) 25.

[18] M.D. Johannes, A.M. Stux, K.E. Swider-Lyons, Phys. Rev. B 77 (2008) (7).

[19] M. Sathiya, K. Ramesha, G. Rousse, D. Foix, D. Gonbeau, A.S. Prakash, M.L. Doublet, K. Hemalatha, J.M. Tarascon, Chem. Mat. 25 (2013) (7) 1121.

[20] G. Ceder, Y.M. Chiang, D.R. Sadoway, M.K. Aydinol, Y.I. Jang, B. Huang, Nature 392 (1998) (6677)

694.

[21] Y.S. Meng, M.E. Arroyo-de Dompablo, Energy \& Environmental Science 2 (2009) (6) 589.

[22] M.K. Aydinol, A.F. Kohan, G. Ceder, K. Cho, J. Joannopoulos, Phys. Rev. B 56 (1997) (3) 1354.

[23] D.M. Ceperley, B.J. Alder, Phys. Rev. Lett. 45 (1980) (7) 566.

[24] J.P. Perdew, J.A. Chevary, S.H. Vosko, K.A. Jackson, M.R. Pederson, D.J. Singh, C. Fiolhais, Phys. Rev. B 46 (1992) (11) 6671.

[25] V.I. Anisimov, J. Zaanen, O.K. Andersen, Phys. Rev. B 44 (1991) (3) 943.

[26] J. Heyd, G.E. Scuseria, M. Ernzerhof, J Chem Phys 118 (2003) (18) 8207.

[27] G. Kresse, J. Furthmuller, Phys. Rev. B 54 (1996) (16) 11169.

[28] M.D. Johannes, K. Hoang, J.L. Allen, K. Gaskell, Phys. Rev. B 85 (2012) (11) 115106.

[29] V.L. Chevrier, S.P. Ong, R. Armiento, M.K.Y. Chan, G. Ceder, Phys. Rev. B 82 (2010) (7).

[30] Y.D. Cho, G.T.K. Fey, H.M. Kao, Journal of Solid State Electrochemistry 12 (2008) (7-8) 815.

[31] C.T. Love, W. Dmowski, M.D. Johannes, K.E. Swider-Lyons, Journal of Solid State Chemistry 184 (2011) (9) 2412.

[32] S. Laubach, S. Laubach, P.C. Schmidt, D. Ensling, S. Schmid, W. Jaegermann, A. Thissen, K.

Nikolowski, H. Ehrenberg, Physical Chemistry Chemical Physics 11 (2009) (17) 3278.

[33] P. Xiao, Z.Q. Deng, A. Manthiram, G. Henkelman, The Journal of Physical Chemistry C 116 (2012) (44) 23201.

[34] C.J. Patridge, C.T. Love, K.E. Swider-Lyons, M.E. Twigg, D.E. Ramaker, Journal of Solid State Chemistry 203 (2013) (0) 134.

[35] M. Sathiya, G. Rousse, K. Ramesha, C.P. Laisa, H. Vezin, M.T. Sougrati, M.L. Doublet, D. Foix, D. Gonbeau, W. Walker, A.S. Prakash, M. Ben Hassine, L. Dupont, J.M. Tarascon, Nat Mater 12 (2013) (9) 827.

[36] S. Sarkar, P. Mahale, S. Mitra, J. Electrochem. Soc. 161 (2014) (6) A934.

[37] D.B. Le, S. Passerini, J. Guo, J. Ressler, B.B. Owens, W.H. Smyrl, J. Electrochem. Soc. 143 (1996) (7)

2099.

[38] K.E. Swider-Lyons, C.T. Love, D.R. Rolison, Solid State Ion. 152 (2002) 99.

[39] R.E. Ruther, H. Zhou, C. Dhital, K. Saravanan, A.K. Kercher, G. Chen, A. Huq, F.M. Delnick, J. Nanda, Chem. Mat. 27 (2015) (19) 6746.

[40] A. Manthiram, J.B. Goodenough, J. Power Sources 26 (1989) (3-4) 403. 